\title{
DIRECTIONAL SENSITIVITY AND MECHANICAL COUPLING DYNAMICS OF CAMPANIFORM SENSILLA DURING CHORD- WISE DEFORMATIONS OF THE FLY WING
}

\author{
BY MICHAEL H. DICKINSON* \\ Department of Zoology NJ-15, University of Washington, Seattle, WA 98195, \\ $U S A$
}

Accepted 14 April 1992

\begin{abstract}
Summary
The complex morphology of an insect campaniform sensillum is responsible for transforming strains of the integument into a displacement of the campaniform dome and subsequently a deformation of the dendritic membrane. In this paper, the first step in this coupling process was investigated in identified campaniform sensilla on the wing of the blowfly by stimulating the sensilla with chord-wise deflections of the wing blade. Campaniform sensilla neurones were sensitive to both dorsal and ventral deflections of the wing, and thus exhibited no strong directional sensitivity to the chord-wise components of wing deformation. These results are consistent with a simplified mechanical model in which the wing veins act as cylinders that undergo bending and torsion during chord-wise wing deformation.

By comparing the responses of campaniform neurones to chord-wise deflections of the wing with those evoked by direct punctate stimulation of the dome, it is possible to estimate the dynamic properties of the coupling process that links wing deformation to dome deformation. In the identified campaniform neurone examined, wing-dome coupling attenuates high frequencies and transforms the chord-wise deflections of the wing into dome deformation similar in degree of excitation to that caused by direct punctate indentions that are two or more orders of magnitude smaller in size.
\end{abstract}

\section{Introduction}

The task of mechanosensory neurones is to translate the mechanical energies that impinge upon body structures into streams of electrical signals that can be interpreted by the cells of the central nervous system. Typically, the first stage of this process is executed by one or a series of coupling structures that transform and

* Present address: Department of Organismal Biology and Anatomy, The University of Chicago, 1025 East 57th Street, Chicago, IL 6063, USA.

Key words: mechanoreceptors, mechanical coupling, fly wings, Calliphora vomitoria. 
conduct mechanical energy to the dendritic surface of the sensory neurone. One example of such a structure is the dome and socket of campaniform sensilla, strainsensitive proprioceptors of the insect cuticle. The complex morphology of each sensillum is responsible for transforming compression and tension in the cuticle surrounding the socket into a small deformation of the dome and ultimately a distortion of the dendritic membrane that produces a transduction current (Chapman et al. 1973; Spinola and Chapman, 1975; Thurm and Küppers, 1980; Barth, 1981). The geometry of the dome and socket also endows elliptical sensilla with directional sensitivity, because of a higher sensitivity to compression perpendicular to their long axis (Thurm et al. 1975; Zill and Moran, 1981). The coupling apparatus of campaniform sensilla can also play an important role in establishing the frequency response of the sensillum, as has been shown for mechanosensory hairs of a cricket (Shimozawa and Kanou, 1984a,b) and a caterpillar (Tautz, 1977a,b).

The role of mechanical coupling in establishing directional sensitivity has been elegantly analyzed for the campaniform sensilla on the relatively stiff cockroach leg (Zill and Moran, 1981). These elliptically shaped campaniform sensilla display a directional sensitivity established by the orientation of their long axis with respect to the tension and compression vectors produced during loading of the cylindrical leg. Extreme axial asymmetry is also correlated with directional sensitivity in the closely packed campaniform fields at the base of the haltere in Calliphora (Thurm et al. 1975). A similar phenomenon is responsible for directional sensitivity in the arachnid analogues of campaniform sensilla, slit sensilla, which have been the subject of a detailed analysis of coupling mechanics (see Barth, 1981, 1984, for reviews). Little is known, however, of the coupling process for sensilla that reside on more flexible and geometrically complex structures such as the wings. Flies bear on their wings a discrete and stereotyped array of campaniform sensilla whose encoding properties have recently been analyzed using direct punctate stimulation (Dickinson, 1990a,b). However, the responses of these sensilla to deformation of the wing and the dynamics that couple wing bending to dome indentation have not previously been quantified.

In this paper, I examine the responses of campaniform sensilla on the wing of the blowfly, Calliphora vomitoria, to chord-wise deformations of the wing. Chordwise deflections occur during flight as the wings develop a positive camber during each half-stroke (Nachtigall, 1979; Ellington, 1984; Ennos, 1989). An interesting feature of these deformations is that they can occur in both directions during the wing beat, since the wing is thought to adopt an appropriate camber for both the upstroke and downstroke. Thus, the first goal in this paper is to examine qualitatively the responses of campaniform neurones to both dorsal and ventral directions of chord-wise wing flexion. Second, in order to examine the contribution of coupling to the dynamic response of the campaniform sensilla, a quantitative measure is made by comparing the neuronal responses elicited by mechanical noise stimuli applied to the wing with those measured during direct punctate stimulation of the sensillum dome. 


\section{Materials and methods \\ Stimulation and recording}

Previously published techniques for recording the responses of wing campaniform sensilla neurones (Dickinson, 1990a) were modified to allow chord-wise deformations of the wing. Fly wings were removed and glued dorsal side up with cyanoacrylate glue to an acrylic recording stage containing two recording wells filled with saline, which made contact with the cut distal and proximal ends of the wing. One side of the stage was cut away parallel to the long axis of the wing so that the second vein and all anterior portions were glued down, while the posterior portion extended unsupported off the edge (Fig. 1). The stimulus probe consisted of a Ling model 201 vibration generator driven by a PID feedback amplifier. The probe position was monitored optically as described previously (Dickinson, $1990 \mathrm{a}$ ). The wing was attached to the probe by means of a small spring-loaded clamp (a modified doll's clothes pin, Archie McPhee, Seattle), which held the posterior portion of the wing firmly near the fourth vein. The optical sensor measured displacements of the probe tip and thus recorded the movements of the wing just posterior to the fourth vein. As the stimulus probe moved up and down, the posterior portion of the wing was translated vertically relative to the fixed anterior portion, eliciting responses from the campaniform sensilla neurones. Upward vertical deflections of the posterior portion of the wing are defined here as dorsal displacements and correspond to concave deformations of the dorsal surface. Deflections in the opposite direction are termed ventral displacements. The nomenclature and map of the identified distal campaniform sensilla have been previously published (Dickinson and Palka, 1987; Dickinson, 1990a).

All the neurones of the third vein displayed some sensitivity to the stimulus and, consequently, it was not possible unambiguously to identify the spikes of individual cells in the extracellular recordings. Consequently, the preparation was typically simplified to localize the stimulus and to allow for unambiguous recordings from just two neurones, the ACV (anterior cross-vein sensillum) and L3-1 (the first sensillum of the third vein), whose spikes are easily distinguished by size (Dickinson and Palka, 1987). Two chord-wise incisions were made from the trailing edge of the wing to the posterior side of the third vein; one distal to L3-1 and the other proximal to the ACV (Fig. 1A). These incisions decreased the amount of tension on the most distal and most proximal portions of the third vein during translation of the posterior half of the wing. In addition, the third vein was crushed distal to the L3-1 sensillum, destroying the axons of the L3-2 and L3-3 sensilla, and the giant sensillum of the radius (GSR) and the dorsal humeral crossvein sensillum (d-HCV) were ablated with a fine razor blade shard. The only sensillum that could potentially contaminate the recordings was the L3-v, whose spikes are rarely detectable in extracellular recordings. Further, by crushing either the ACV or the L3-1 sensillum with forceps, it was possible to record the response of the other neurone unambiguously.

At the start of each experiment, the vertical offset of the stimulus probe was 


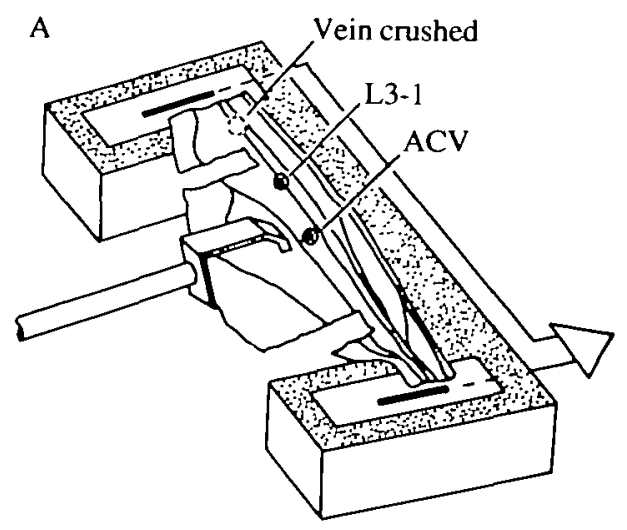

B
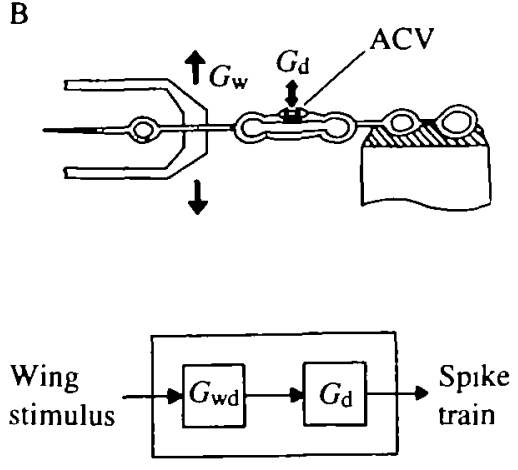

Fig. 1. Preparation used to study the responses of campaniform sensilla to chord-wise deformations of the wing and linear systems model. (A) The anterior half of a Calliphora vomitoria wing is glued with cyanoacrylate adhesive to an acrylic stage. Electrical contact with the veins is made by saline wells at distal and proximal ends of the wing. To record unambiguously the responses of the ACV and L3-1 neurones, the third vein is crushed distal to the L $3-1$ and proximal sensilla are ablated with a razor blade shard. To localize the stimulus and minimize tension acting on the distal portion of the third vein, two incisions are made in the wing, one at the level of the L3-v and another just distal to the L3-1. (B) Detail of stimulus arrangement and method for calculation of wing-dome coupling. (Top) A schematic slice through the wing at the level of the ACV sensillum. Note that the anterior cross vein runs perpendicular to the long axis of the wing. The first and second veins are glued to the recording stage, and the stimulus clamp holds the wing between the fourth and fifth veins. The entire encoding process, $G_{\mathrm{w}}$, is modelled as a cascade of two linear functions, $G_{\mathrm{wd}}$, which accounts for the conversion of wing deformation to dome deformation, and $G_{\mathrm{d}}$, which represents the transformation from dome deformation to spike code (below). Both $G_{w}$ and $G_{\mathrm{d}}$ can be measured using noise stimuli applied to the wing and dome, respectively, with the assumption that punctate indentation is a reasonable substitute for the naturally occurring deformation of the dome. The unknown function $G_{w d}$, wing-dome coupling, is computed from the ratio of $G_{\mathrm{w}} / G_{\mathrm{d}}$ in the frequency domain.

positioned to correspond to the resting, undeformed state. This was done by eye through a dissecting microscope and by monitoring the spike discharge of the tonically active L3-1 neurone, which is excited by both dorsal and ventral deflections and shows no response when the wing is in the resting position. The campaniform sensilla neurones were stimulated with either dorsal or ventral trapezoidal deflections ( $1 \mathrm{~ms}$ rise time) or noise stimuli $(20-400 \mathrm{~Hz}$ bandwidth). The offset and the magnitude of the stimuli could be varied independently.

\section{Analytical methods}

The complete coupling mechanism that transforms wing deflection to strain of 
the dendritic membrane may be divided into two sequential components. The mechanics that link wing deformation to dome deformation are defined as wing-dome coupling and the process that transforms dome indentation into membrane strain as dome-neurone coupling. The net coupling behaviour, wingneurone coupling, which precedes the physiological response of the neurone, is modelled here as a simple cascade of these two processes.

Although it is difficult to measure the separate components of coupling directly, the first element in the cascade, wing-dome coupling, can be measured by the techniques described below. Both the gain and phase of the linear function describing the spike response of a campaniform neurone to a noise stimulus, applied either to the wing or directly to the dome, may be computed from the prespike stimulus average (Dickinson, $1990 a$ ). The functions $G_{\mathrm{d}}(t)$ and $G_{\mathrm{w}}(t)$, where $t$ is time, represent linear response functions calculated from direct punctate stimulation of the dome and chord-wise deformation of the wing respectively (Fig. 1B). The linear model of wing-dome coupling is represented as $G_{\mathrm{wd}}(t)$. In the frequency domain, these three functions are related in a simple manner:

$$
G_{\mathrm{wd}}(j \omega)=G_{\mathrm{w}}(j \omega) / G_{\mathrm{d}}(j \omega),
$$

where $j$ is $\sqrt{-1}$ and $\omega$ is frequency.

Thus, the dynamic behaviour of wing-dome coupling is computed by the ratio of the linear response functions measured using wing deformation and punctate indentation. Because proper stimulation using direct punctate indentation requires that the wing be glued firmly to the recording chamber, and the life time of the excised wings is approximately $20 \mathrm{~min}$, both calculations could not easily be made from individual sensilla. Instead, $G_{\mathrm{w}}(t)$ and $G_{\mathrm{d}}(t)$ functions were measured from the ACV neurone in separate preparations. The data from punctate stimulation (15 experiments) have been previously published in another form (Dickinson, 1990a). In those experiments it was possible to calculate the actual indentation of the dome relative to the surrounding vein cuticle by careful measurement of the campaniform dome, socket and stimulus probe compliances. The magnitudes of the wing deflection stimuli were chosen to produce a mean spike rate that closely matched that elicited during punctate stimulation. The results of the dome and wing stimulation experiments were averaged after transformation to the frequency domain. The ratio $G_{\mathrm{w}}(j \omega) / G_{\mathrm{d}}(j \omega)$ was then computed to yield the transfer function that describes wing-dome coupling. The phase introduced by this coupling process was found from the difference of the phases calculated from $G_{\mathrm{w}}$ and $G_{\mathrm{d}}$.

\section{Results}

\section{Response polarity}

The rapidly adapting ACV neurone was responsive to both dorsal and ventral deformations of the wing. However, as shown in Fig. 2 (left-hand column), the polarity of the deflections to which the neurones responded was strongly 

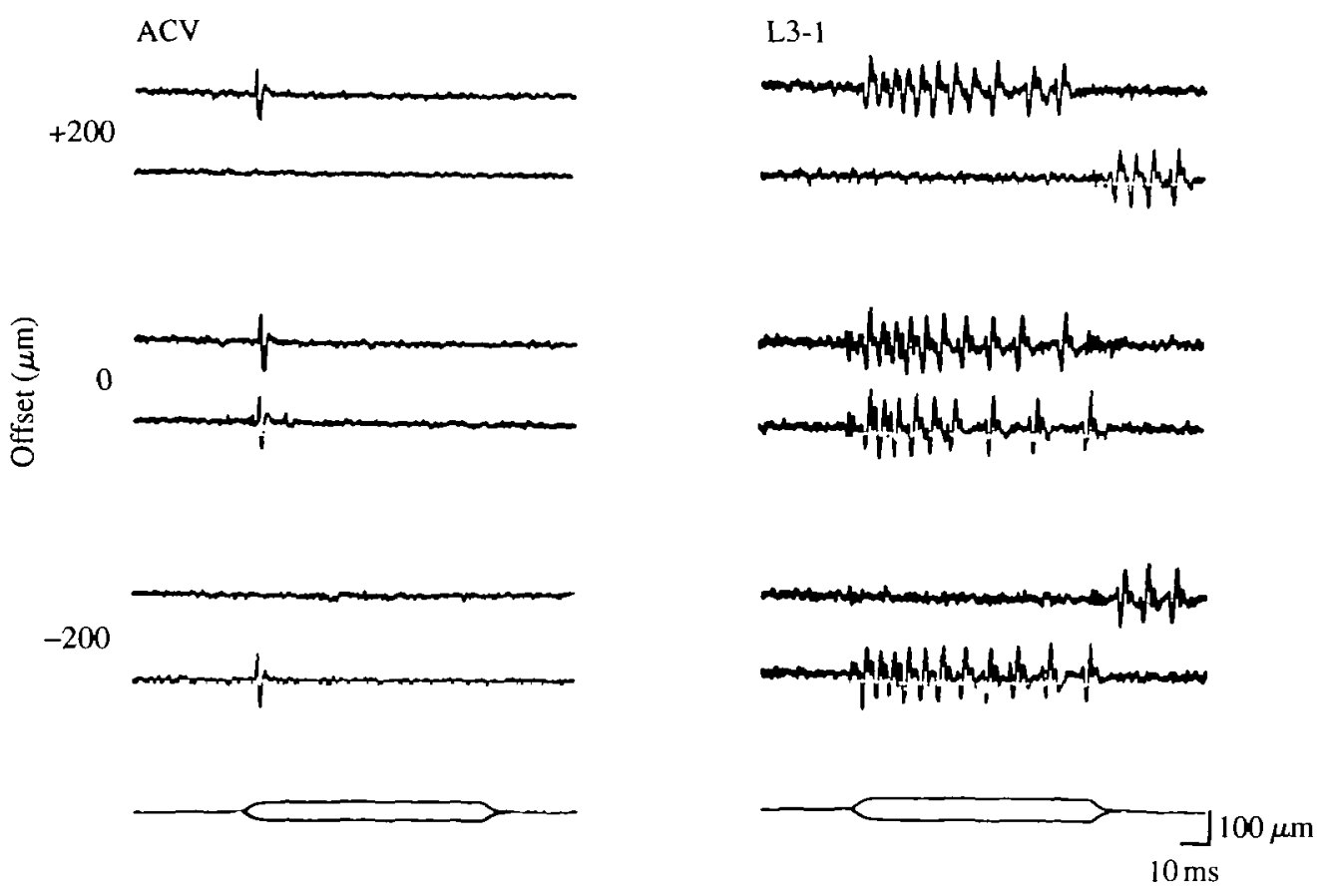

Fig. 2. Response of ACV and L3-1 neurones to direction and mean offset of chordwise wing deformations. Both left and right panels consist of three pairs of traces recorded with the mean offset values shown at the left (in $\mu \mathrm{m}$, positive values indicate dorsal deflection). The stimuli, consisting of trapezoidal deflections, are indicated at the bottom of each panel. The top and bottom traces in each pair of recordings show the responses to dorsal and ventral deflections, respectively. With no stimulus offset, the ACV and L3-1 neurones respond to both dorsal and ventral stimuli and display no obvious directional sensitivity. When an offset is imposed upon the wing, the neurones respond only when the wing is deflected further from the resting, undeformed position. The L3-1 displays an off-response at the end of stimuli that deflect the wing towards the resting position.

dependent upon the stimulus offset. If the posterior portion of the wing was bent dorsally, the ACV responded at the onset of trapezoidal deflections that moved the wing to a more extreme dorsal position. If a ventral offset was imposed on the wing, then the neurone responded to further ventral deflections. With no offset, the neurones responded to both dorsal and ventral deformations. The ACV typically responded to trapezoidal deformations of the wing with a single spike at the onset of the stimulus. However, a small burst of spikes at the onset and an offresponse were sometimes seen, especially in response to stimuli with larger magnitudes.

The tonically responding L3-1 neurone displayed a directional response similar to the ACV cell (Fig. 2, right-hand column). However, a background spike frequency was present in the L3-1 whenever the wing was offset from the resting position. In these experiments, the L3-1 neurone was allowed to adapt for several 


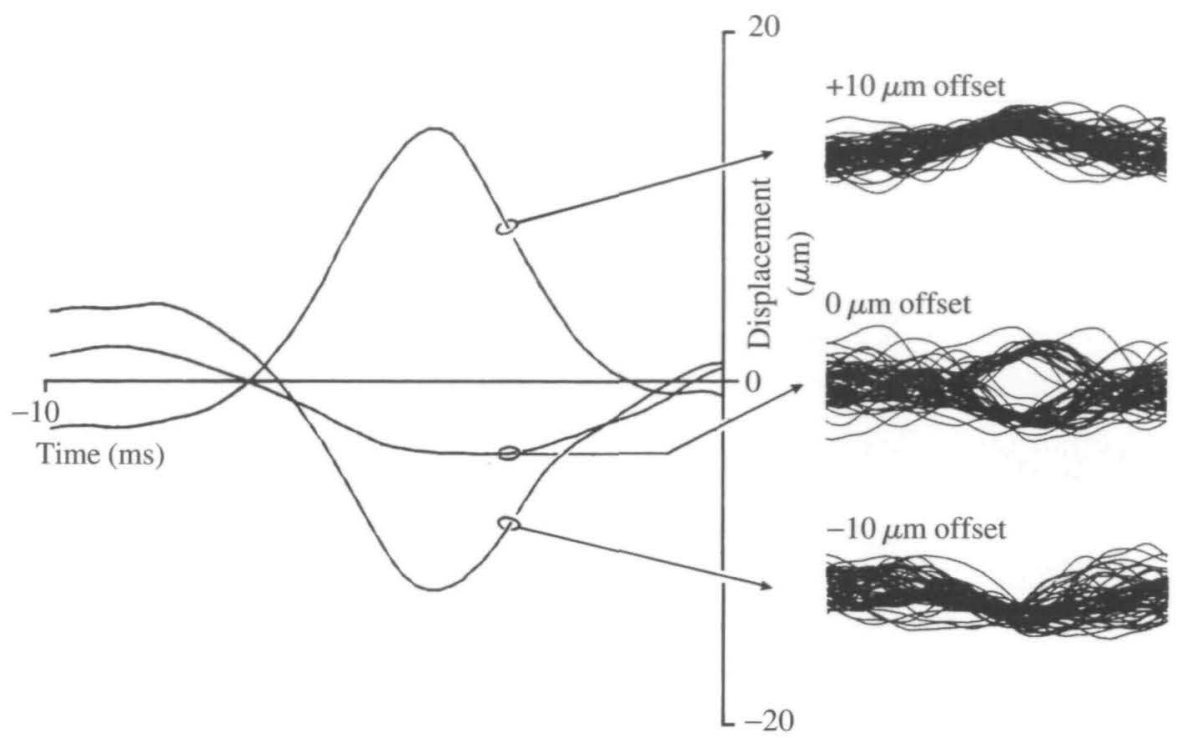

Fig. 3. Noise stimuli that elicit responses of an L3-1 neurone applied at different levels of mean wing deflection. The three traces on the left show the pre-spike stimulus average computed from $10 \mathrm{~ms}$ noise segments preceding 100 spikes during a $2.5 \mathrm{~s}$ stimulation of the wing. Fifty individual stimulus traces preceding spike occurrences are displayed to the right. The mean level of wing deflection is indicated at the left of the superimposed traces (in $\mu \mathrm{m}$, positive values indicate dorsal deflections). When the noise stimulus was added to a mean dorsal deflection, the neurone responded to further dorsal deformations. However, if a mean ventral deflection was imposed, the neurone responded only to ventral transients of the noise stimulus. With no stimulus offset, the cell responded to both dorsal and ventral deflections, as clearly indicated by the bimodal distribution of stimulus events preceding each spike occurrence.

minutes to any imposed steady offset until the spike rate was near zero. The spike frequency increased with trapezoidal stimuli that deflected the wing further from the undeflected position. In contrast, if the wing was bent towards the undeflected position, the cell displayed an off-response at the end of the stimulus. This offresponse was a characteristic feature of the two other tonic neurones (L3-3, L3-v) on the third vein (data not shown).

The effects of stimulus offset on the directional response of the campaniform sensilla neurones were also manifest when the wing was stimulated with mechanical noise. Fig. 3 (left) shows the averaged time course of the stimulus preceding spike occurrences in an L3-1 neurone, measured at three different levels of stimulus offset. Superpositions of individual stimulus traces preceding spike occurrences are shown to the right of each averaged trace. When the probe was positioned to produce a net ventral deformation of the wing, the L3-1 responded to further ventral deflections. Yet when the noise stimulus was offset with a dorsal deformation, the same campaniform sensillum responded to further dorsal deflections. With no net stimulus offset, the neurone responded to both dorsal and 
ventral transients in the noise stimulus, resulting in a pre-stimulus average that nearly cancelled out.

\section{Dynamics of wing-dome coupling}

Fig. 4A compares the frequency response functions measured with direct punctate and whole-wing stimulation. The data from six wing stimulus experiments and 15 dome stimulus experiments were averaged separately and used to construct the frequency response of wing-dome coupling. Because the ACV neurone was responsive to both dorsal and ventral deflections it was necessary to apply a small offset to the noise stimulus to ensure that the sensilla responded to wing movements of one polarity only. The results of three experiments with dorsal offset and three with ventral offset revealed similar frequency responses, and the data from all six experiments were pooled.

The calculated gain and phase of wing-dome coupling are shown in Fig. 4B. Wing-dome coupling attenuated high frequencies, yet had little effect on the phase response. At $150 \mathrm{~Hz}$, the typical wing-beat frequency of Calliphora, a given displacement of the posterior end of the anterior cross vein resulted in an excitation of the campaniform neurone equivalent to that produced by a direct
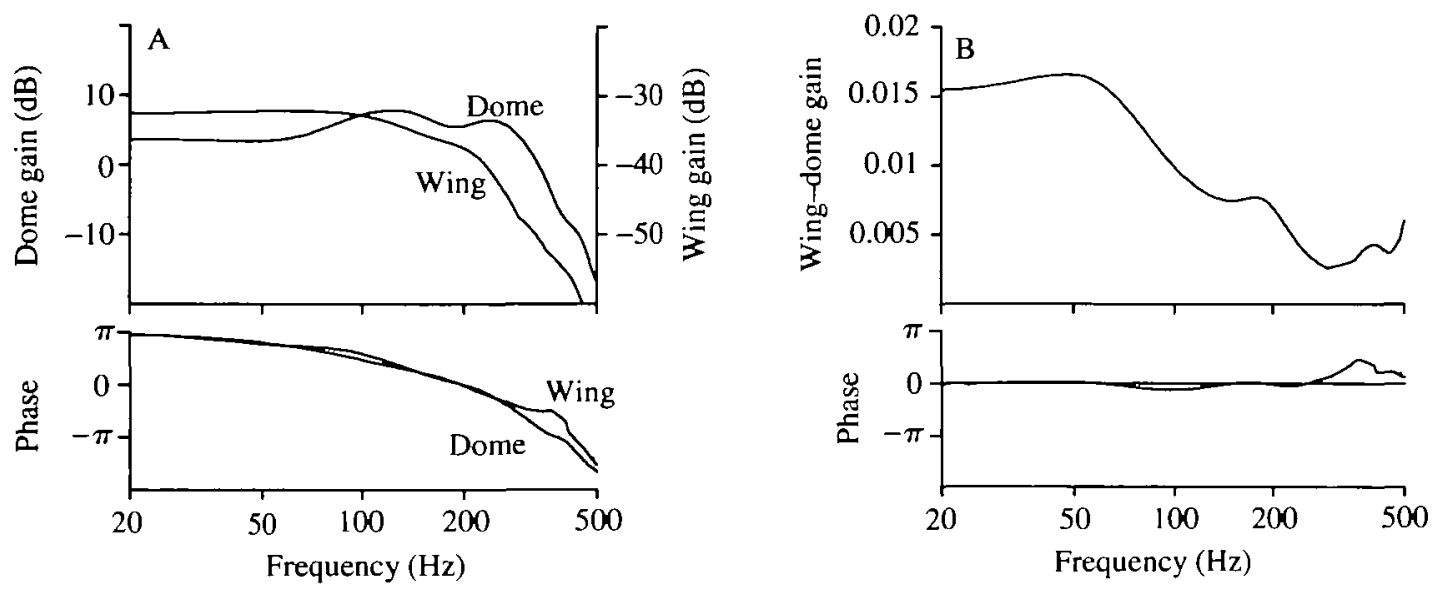

Fig. 4. Comparison of frequency responses of the ACV neurone calculated with direct punctate indentation and chord-wise wing deformation. (A) The gain and phase relationships calculated from punctate indentation experiments and wing deformation experiments are superimposed. For both functions, the gain (in spikes $\mathrm{s}^{-1} \mathrm{~nm}^{-1}$ ) has been plotted on a decibel scale. The data are the mean values from several experiments (wing stimulation, $N=6$; dome stimulation, $N=15$ ). The dome stimulation data have been previously published in another form (Dickinson, 1990b). The gain measured with deformation of the wing shows a greater attenuation at high frequencies. (B) The two frequency responses in A were used to calculate the gain and phase response of the wing-dome coupling process (see Materials and methods). The coupling is a unitless function since it is calculated from the ratio of two functions with identical units. 
punctate indentation approximately 100 times smaller in magnitude. Thus, a $1 \mu \mathrm{m}$ displacement of the fourth vein would result in deformation equivalent in level of excitation to the response elicited by a $10 \mathrm{~nm}$ indentation of the campaniform dome.

\section{Discussion}

Lack of directional sensitivity among wing campaniform sensilla

The responses of the ACV and L3-1 neurones elicited by chord-wise deflections of the wing are similar to those produced with direct punctate indentation of the dome (Dickinson and Palka, 1987). The ACV neurone adapts extremely rapidly to step deflections of the wing, whereas the L3-1 continues to fire for the duration of the stimulus. In the absence of a mechanical offset, neither the ACV nor the L3-1 neurone exhibit a strong directional sensitivity to the polarity of chord-wise wing deformation (Fig. 2). During maintained deformations, the campaniform sensilla neurones responded to stimuli that increased the deflection of the wing and not to those that moved the wing towards the undeflected position. In this latter case, however, the L3-1 neurone typically displayed an off-response burst after the termination of the stimulus.

The similarity in the responses of the fly wing neurones to both dorsal and ventral flexion must result from the interaction of two factors: the strain distribution on the wing during deformation and the intrinsic directional sensitivities of the sensilla. A simple prediction of the wing strain, in which the anterior cross vein and the third vein are modelled as orthogonal hollow cylinders is shown in Fig. 5 and is based on well-characterized solutions of the stress distributions for this geometry (Beer and Johnston, 1981). An upward deflection of the wing (Fig. 5A), would result in compression forces on the dorsal surface of the cross vein acting parallel to the vein axis, with tension forces acting perpendicularly around the circumference of the vein. The cross vein also transmits the upward displacement to the third vein, thereby creating orthogonal helices of compression and tension forces that are maximal at an angle of $45^{\circ}$ with respect to the long axis of the vein. During downward flexion of the wing (Fig. 5B), the dorsal surface of the cross vein would undergo tension axially with compression forces oriented circumferentially, and the maximum compression and tension helices on the third vein would rotate by $90^{\circ}$. According to this model, the neurones of the campaniform sensilla could only respond to both upward and downward wing flexion if they display little or no intrinsic directional sensitivity. That is to say, the model requires that the sensilla respond equally to compression forces acting from orthogonal directions. Gnatzy et al. (1987), who examined the ultrastructure of the campaniform sensilla in the closely related Calliphora vicina, found that the six most distal campaniform sensilla on the fly wing, including the ACV and L3-1 sensilla, possess dendrites and tubular bodies that are circular in cross section. Thus, these sensilla lack the flattened dendritic morphology characteristic of 

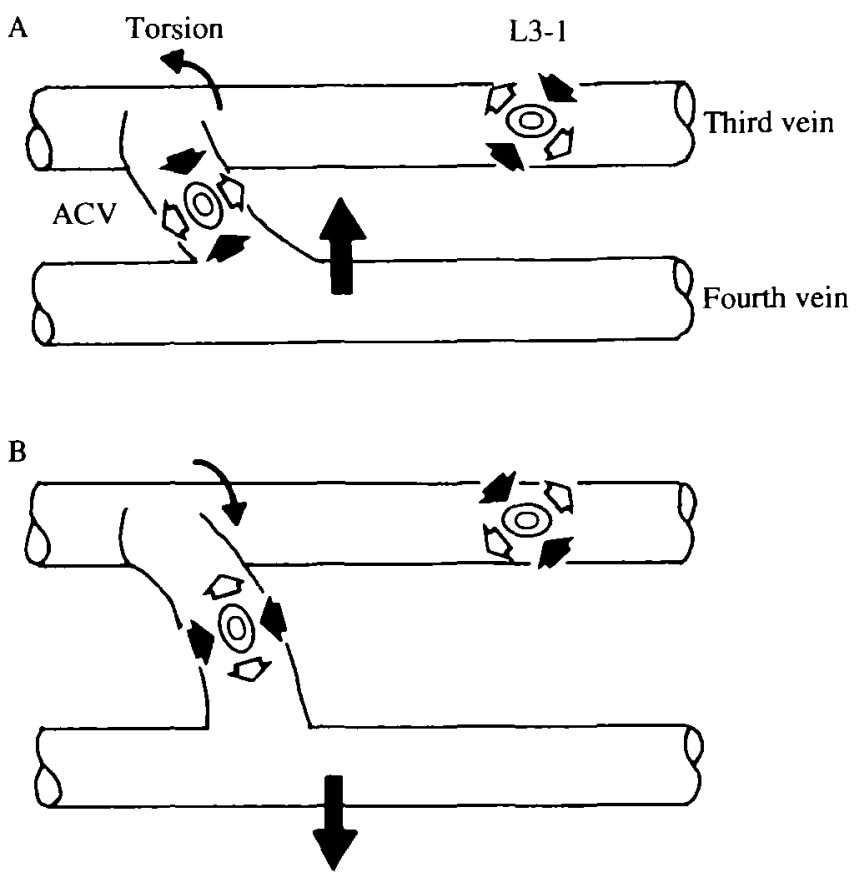

Fig. 5. Model of forces acting on campaniform sensilla during chord-wise flexion of the wing. The diagram shows the predicted distribution of compression (solid arrows) and tension (open arrows) acting on the ACV and L3-1 sensilla. (A) During upward flexion of the fourth vein, the cross vein would undergo simple upward bending, resulting in an axial compression of the ACV sensilla and circumferential tension. The upward movement of the cross vein would transmit a torsional force acting on the third vein, resulting in orthogonal helices of compression and tension. (B) A downward displacement of the fourth vein results in a reversal of the compression and tension vectors acting on both sensilla.

directionally sensitive sensilla, and it is certainly plausible that they could respond equally well to compression stimuli with orthogonal orientations.

Although the dendrite of campaniform sensilla neurones is circular, the dome and socket are slightly elliptical in outline (Gnatzy et al. 1987; Dickinson, 1989), with the long axes oriented parallel to the veins as indicated in Fig. 5. Such morphology would tend to resist compression oriented parallel to the wing veins, thus endowing the sensilla with directional sensitivity. This arrangement would not alter the response of the L3-1 neurone to upward and downward wing displacements, because in both cases compression forces would be acting obliquely to the long axis. However, the ACV neurone would be expected to display less sensitivity to upward flexion because the compression forces are parallel to the long axis of the sensillum. However, according to linear elastic theory, the compression forces acting along the parallel axis during an upward flexion will be greater than the compressive forces acting circumferentially during a downward flexion of equal 
magnitude. Therefore, it is possible that the elliptical morphology of the ACV dome and socket might function to counteract the directional bias that results from the expected asymmetry in strain magnitudes, thus endowing this sensillum with similar sensitivities to upward and downward wing flexion.

The above model oversimplifies the geometry of the wing, and the actual stress distributions are likely to be more complex than those shown in Fig. 5. For example, the interior morphology of the anterior cross vein has been likened to a vacuum cleaner hose (Wisser, 1987), consisting of thick rings of cuticle, separated by narrow segments of thinner, presumably more flexible, cuticle. In addition, the model ignores the wing membrane that must act in some degree to transmit forces from one vein to another. Nevertheless, the simplified model is consistent with the ultrastructure of the campaniform sensilla and represents a parsimonious explanation of the physiological results.

By whatever mechanical or physiological means it is achieved, the lack of directional sensitivity presents a potential source of processing error for the central nervous system (CNS), because the afferent spike trains produced by a single sensillum during dorsal and ventral flexion could be identical. There are several plausible means by which the CNS could interpret these potentially ambiguous signals. During flight, wing deformation is complex, consisting of length-wise, chord-wise and torsional components (Wooton, 1981; Ellington, 1984; Ennos, 1989). It is possible that the responses of the campaniform sensilla to the net pattern of wing flexion are less directionally ambiguous. Further, the kinematics of wing deformations during flight, although variable, may be stereotyped enough for directionality not to be important. For example, the torsional wave generated during supination is usually much larger than that during pronation, and the CNS might be wired such that it always interprets campaniform activity during flight as an indication of supination. This strategy would be facilitated by the fact that each campaniform sensilla neurone probably fires only one action potential during each wing-beat cycle (Dickinson, 1990a). Finally, although the response of a single neurone may be ambiguous, the CNS might be able to derive the direction of wing flexion from the population response of all the campaniform sensilla neurones and mechanoreceptors from other qualities. For example, the wing base contains a large number of small campaniform sensilla that appear to be well placed to encode the position of the wing with respect to the thorax. The activity of the distal campaniform sensilla during pronation and supination could be distinguished by the CNS through comparison with this independent monitor of wing position.

\section{Dynamics of the wing-dome coupling process}

A comparison of the frequency responses of the ACV neurone measured with chord-wise deformation and punctate stimulation indicates that wing-dome coupling attenuates high frequencies (Fig. 4B). Thus, this initial stage of mechanical coupling acts as a low-pass filter-on wing deformations. The attenuation of high frequencies may result from filtering behaviour residing in the mechanical and geometrical properties of the wing, most probably due to the viscoelastic 
properties of the vein cuticle, underlying epithelial cells and internal haemolymph. As argued more fully elsewhere (Dickinson, 1990a), it is likely that the ACV and the other campaniform neurones of the wing each fire a single action potential at a precise phase in the wing-beat cycle. Because the low-pass cut-off is near the wingbeat frequency in Calliphora $(150 \mathrm{~Hz})$, this filtering will further decrease the probability that the neurone will fire more than once during each wing stroke.

One task of the mechanical coupling process is to transform the relatively large deformation of the cuticle into a much smaller deformation of the dome. However, it is not clear how the coupling apparatus of a campaniform sensillum transforms strain in the surrounding cuticle into a deformation of the dome and excitation of the underlying sensory dendrite. Spinola and Chapman (1975) argued that a naturally occurring stimulus results in an indentation of the dome, on the basis of experiments in which cockroach tibial campaniform sensilla were stimulated simultaneously with leg deformation and direct punctate indentation. However, on the basis of a detailed analysis of ultrastructure and by analogy with the well-characterized arachnid slit sensilla, Barth (1981) has argued that an outward bulging of the dome is the adequate stimulus during cuticle deformation. Because of the experimental difficulties with direct elevation of the campaniform dome, I have used punctate indentation as a means of transmitting a mechanical stimulus directly to the campaniform dome, bypassing the first stage of stimulus transmission. I must explicitly assume, therefore, that the dynamics of deformation revealed by direct punctate stimulation are not fundamentally different from those occurring naturally, irrespective of the actual direction or form of dome indentation.

Given the above assumptions, displacement of the third vein of the fly wing relative to the fourth vein results in an excitation that is equivalent in magnitude to that produced by a direct punctate indentation of approximately $1 / 100$ the size (Fig. 4B). This coupling factor is likely to be nonlinearly related to the magnitude of wing deformation, and the above value, calculated from experiments using small-amplitude wing deflections, probably underestimates the degree of attenuation during large deformations. Unlike campaniform sensilla positioned on stiff cylinders such as cockroach legs, the wing campaniform sensilla reside on a relatively flexible plate. The magnitude of the wing deformations that are encountered during flight or grooming are quite large relative to those expected on the much less compliant legs during walking. The mechanical properties responsible for the appropriate transformation of cuticle deformation into dome indentation must vary according to location to ensure that the strains produced during flight and other activities result in dome deformations of the appropriate magnitude.

I wish to thank Andy Biewener, John Palka, Tom Daniel and Bob Pinter for their comments and suggestions with this project. This work was supported by a NSF Graduate Fellowship, and NSF Grant BNS-8507460 to John Palka. 


\section{References}

Barth, F. G. (1981). Strain detection in the arthropod exoskeleton. In Sense Organs (ed. M. S. Laverack and D. J. Cosens), pp. 112-142. London: Blackie.

BARTH, F. G. (1984). Slit sensilla and the measurement of cuticular strains. In Neurobiology of Arachnids (ed. F. G. Barth), pp. 162-188. Berlin: Springer Verlag.

BeER, F. P. AND Johnston, E. R. (1981). Mechanics of Materials. New York: McGraw-Hill.

Chapman, K. M., Duckrow, R. B. and Moran, D. T. (1973). Form and role of deformation in excitation of an insect mechanoreceptor. Nature 197, 699-701.

Dickinson, M. H. (1989). The encoding properties of campaniform sensilla on the dipteran wing blade. PhD dissertation, University of Washington.

Dickinson, M. H. (1990a). Linear and nonlinear encoding properties of an identified mechanoreceptor on the fly wing measured with mechanical noise stimuli. J. exp. Biol. 151, 219-244.

Dickinson, M. H. (1990b). Comparison of encoding properties of campaniform sensilla on the fly wing. J. exp. Biol. 151, 245-261.

Dickinson, M. H. AND PALKA, J. (1987). Physiological properties, time of development, and central projections are correlated in the wing mechanoreceptors of Drosophila. J. Neurosci. 7, 4201-4208.

Ellington, C. P. (1984). The aerodynamics of hovering insect flight. III. Kinematics. Phil. Trans. R. Soc. Lond. B 305, 41-78.

Ennos, A. R. (1989). The kinematics and aerodynamics of the free flight of some Diptera. J. exp. Biol. 142, 49-85.

Gnatzy, W., Grunert, U. and Bender, M. (1987). Campaniform sensilla of Calliphora vincina (Insecta, Diptera). I. Topography. Zoomorphology 106, 312-319.

NaChTigall, W. (1979). Rasche Richtungsănderungen und Torsionen schwingender Fliegenflügel und Hypothesen über zugeordnete instationäre Strömungseffekte. J. comp. Physiol. A 139, 351-355.

ShimozaWA, T. AND KANOU, M. (1984a). The aerodynamics and sensory physiology of range fractionation in the cercal filiform sensilla of the cricket Gryllus bimaculatus. J. comp. Physiol. 155, 495-505.

Shlmozawa, T. and Kanou, M. (1984b). Varieties of filiform hairs, range fractionation by sensory afferents and cercal interneurones of a cricket. J. comp. Physiol. 155, 485-493.

Spinola, S. M. and Chapman, K. M. (1975). Proprioceptive indentation of the campaniform sensilla of cockroach legs. J. comp. Physiol. 96, 257-272.

TAUTZ, J. (1977a). Reception of medium vibration by thoracal hairs of caterpillars of Barthra brassicae L. (Lepidoptera, Noctuidae). I. Mechanical properties of the receptor hairs. J. comp. Physiol. 118, 13-31.

TAUTZ, J. (1977b). Reception of medium vibration by thoracal hairs of caterpillars of Barthra brassicae L. (Lepidoptera, Noctuidae). II. Response characteristics of the sensory cell. J. comp. Physiol. 125, 67-77.

Thurm, U. AND KÚpPERS, J. (1980). Epithelial physiology of insect sensilla. In Insect Biology in the Future (ed. M. Locke and D. Smith), pp. 735-763. New York: Academic Press.

Thurm, U., Stedtler, A. ANd Foelix, R. (1975). Reizwirksame Verformungen der Terminalstrukturen eines Mechanorezeptors. In Verhandlungsberichte der Deutschen Zoologischen Gesellschaft 1974 (67), pp. 37-41. Stuttgart: Gustav Fischer Verlag.

WISSER, A. (1987). Mechanisms of wing rotating regulation in Calliphora erythrocephala (Insecta, Diptera). Zoomorphol. 106, 261-268.

Wooton, R. J. (1981). Support and deformability of insect wings. J. Zool., Lond. 193, 447-468.

ZILL, S. N. AND MORAN, D. T. (1981). The exoskeleton and insect proprioception. I. Responses of tibial campaniform sensilla to external and muscle-generated forces in the American cockroach, Periplaneta americana. J. exp. Biol. 91, 1-24. 
